\title{
Comité de Protection des Personnes
}

\author{
Nathalie Brion, ${ }^{1}$ Jean-Paul Demarez, ${ }^{2}$ Chantal Belorgey ${ }^{3}$ et les participants à la Table Ronde $n^{\circ} 1$ \\ de Giens $X^{*}$ \\ 1 Centre Hospitalier de Versailles, Le Chesnay, France \\ 2 Pierre Fabre Médicaments, Boulogne, France \\ 3 Agence française de sécurité sanitaire des produits de santé (Afssaps), Saint-Denis, France
}

\section{Résumé}

\begin{abstract}
La transposition de la directive 2001/20/CE relative aux bonnes pratiques cliniques dans la conduite des essais de médicaments à usage humain a nécessité la modification de certaines dispositions du droit français en matière de protection des personnes se prêtant à des recherches biomédicales, notamment celles relatives aux conditions d'autorisation des recherches biomédicales. Le régime déclaratif à l'autorité compétente antérieur devient un régime d'autorisation préalable, et l'avis préalable d'un comité de protection a désormais la nécessité d'être expressément favorable pour que l'essai soit mis en œuvre. Des propositions sont formulées par cette table ronde afin de favoriser la stabilité et le professionnalisme des Comités de Protection des Personnes (CPP). Mots clés : Comité de Protection des Personnes (CPP), recherche biomédicale, essais cliniques, législation
\end{abstract}

La loi 88-1138 du 20 décembre $1988,{ }^{[1]} 1$ relative à « la protection des personnes se prêtant à des recherches biomédicales » a mis en place une structure nouvelle en droit de la santé, le Comité Consultatif de Protection des Personnes dans la Recherche Biomédicale (CCPPRB).

La transposition en droit interne de la directive 2001/20/CE ${ }^{[2]} \mathrm{du} 4$ avril 2001, concernant « le rapprochement des dispositions législatives, réglementaires et administratives des Etats-membres relatives à l'application de bonnes pratiques cliniques dans la conduite d'essais cliniques de médicaments à usage humain » a donné au législateur l'occasion de modifier sensiblement ce dispositif législatif dit Loi Huriet-Sérusclat, notamment en ce qui concerne le comité indépendant ci-dessus évoqué.

La loi 2004-806 du 9 août 2004 ${ }^{[3]}$ organise des Comités de Protection des Personnes (CPP), dont l'avis relatif à un projet de recherche biomédicale doit obligatoirement être favorable pour que ce projet puisse être mis en œuvre. Parallèlement, la procédure administrative préalable de déclaration d'intention de recherche relevant du promoteur d'une recherche biomédicale est remplacée par une autorisation de l'autorité administrative compétente.
La loi comporte des modifications des responsabilités, des rôles et de la composition du comité. Celui-ci donne en particulier un «avis conforme » découlant de missions déterminées par la loi. L'avis peut faire l'objet d'une contestation.

Cette table ronde (dont le titre exact était « Comité de Protection des Personnes : Rôle vis-à-vis des promoteurs, des autorités compétentes et des patients ; Organisation dans le cadre des essais cliniques de médicaments ») avait pour but d'examiner ces différents aspects, en se limitant à la seule situation des essais cliniques de médicaments. Elle se situait dans la période séparant la publication de la loi du 9 août $2004^{[3]}$ de celle de ses décrets d'application. Le présent compte rendu ne vise pas à rapporter la teneur de nos débats, mais d'en expliciter la synthèse et les conclusions présentées en séance plénière, après validation par l'ensemble des membres participant à la table ronde.

\section{Travaux préparatoires}

Préalablement à la tenue de cette table ronde, lors des $\mathrm{XX}^{\text {èmes }}$ journées de Giens (du 3 au 5 octobre 2004), des réunions avaient été organisées, les membres pressentis pour participer s'étant

\footnotetext{
* Pour la liste des participants, voir en fin d'article.

1 Toutes les références à la législation peuvent être trouvées sur le site http//www.legifrance.gouv.fr.
} 
répartis en quatre sous-groupes chargés d'examiner plus particulièrement un aspect du sujet proposé.

- Sous-groupe 1 : Rôle du CPP avant la mise en place d'une recherche biomédicale;

- Sous-groupe 2 : Rôle du CPP pendant et après une recherche biomédicale ;

- Sous-groupe 3 : Responsabilités du CPP ;

- Sous-groupe 4 : Organisation du CPP.

Outre leur expérience relative aux CCPPRB, les membres de chacun de ces sous-groupes disposaient de textes de référence :

- la directive européenne 2001/20/CE du 4 avril 2001, ${ }^{[2]}$ cidessus évoquée ;

- la loi 2004-806 du 9 août $2004^{[3]}$ modifiant la Loi HurietSérusclat

- les indications détaillées émanant de la Commission européenne dites Detailed guidance for the request for authorisation of a clinical trial on a medicinal product for human use to the competent authorities, notification substantial amendments and declaration of the end of the trial (version avril 2004); ;4]

- les indications détaillées émanant de la Commission européenne dites Detailed guidance on the application format and documentation to be submitted in an application for an Ethics Committee opinion on the clinical trial on a medicinal product for human use (version avril 2004). [5]

Afin de tenter de prévenir toutes digressions postérieures, le caractère limitant du titre a été souligné. Il s'agissait bien de se borner à la situation de l'essai clinique, ainsi définie dans la directive européenne sus-citée comme «toute investigation menée chez l'homme, afin de déterminer ou de confirmer les effets cliniques, pharmacologiques et/ou les autres effets pharmacodynamiques d'un ou de plusieurs médicaments expérimentaux, et/ou de mettre en évidence tout effet indésirable d'un ou de plusieurs médicaments expérimentaux, et/ou d'étudier l'absorption, la distribution, le métabolisme et l'élimination d'un ou de plusieurs médicaments expérimentaux, dans le but de s'assurer de leur innocuité et/ou efficacité ».

L'expression « recherche biomédicale » en droit français recouvre, en effet, un champ beaucoup plus large de l'activité scientifique que l'essai clinique de médicament.

Dans la situation de l'essai clinique, l'autorité compétente est l'Agence française de sécurité sanitaire des produits de santé (Afssaps).

Rappelons que, au sens de la directive, un « essai non interventionnel » ne constitue pas un essai clinique mais une «étude dans le cadre de laquelle les médicaments sont prescrits de la manière habituelle, conformément aux conditions fixées dans l'autorisation de mise sur le marché (AMM). L'affectation du patient à une stratégie thérapeutique donnée n'est pas fixée à l'avance par un protocole d'essai, elle relève de la pratique courante et la décision de prescrire le médicament est clairement dissociée de celle d'inclure le patient dans l'étude. Aucune procédure supplémentaire du diagnostic ou de surveillance ne doit être appliquée aux patients et des méthodes épidémiologiques sont utilisées pour analyser les données recueillies ».

\section{Examen de la situation des Comités de Protection des Personnes (CPP)}

\subsection{Rôle avant l'essai}

Avant de réaliser une recherche biomédicale sur l'être humain, le promoteur est tenu d'en soumettre le projet à l'avis de l'un des CPP compétents pour le lieu où l'investigateur (ou, le cas échéant, l'investigateur coordonnateur) exerce son activité. Le promoteur ne peut solliciter qu'un seul avis par projet de recherche (article L1123-6 du Code de la santé publique $\left.[\mathrm{CSP}]^{[6]}\right)$. Le comité rend son avis sur les conditions de validité de la recherche (article L1123-7 $\mathrm{CSP}^{[6]}$ ), notamment au regard de :

- «la protection des personnes, notamment la protection des participants ;

- l'adéquation, l'exhaustivité et l'intelligibilité des informations écrites à fournir ainsi que la procédure à suivre pour obtenir le consentement éclairé, et la justification de la recherche sur des personnes incapables de donner leur consentement éclairé ;

- la nécessité éventuelle d'un délai de réflexion ;

- la nécessité éventuelle de prévoir, dans le protocole, une interdiction de participer simultanément à une autre recherche ou une période d'exclusion ;

- la pertinence de la recherche, le caractère satisfaisant de l'évaluation des bénéfices et des risques attendus et le bienfondé des conclusions ;

- l'adéquation entre les objectifs poursuivis et les moyens mis en œuvre ;

- la qualification du ou des investigateurs ;

- les montants et les modalités d'indemnisation des participants ;

- les modalités de recrutement des participants ;

- l'éventualité de constitution d'un comité de surveillance indépendant, à l'initiative du promoteur, et dont ce dernier doit motiver la présence ou l'absence. » Le Comité s'assure également que : 
- le lieu où la recherche est envisagée dispose le cas échéant de l'autorisation administrative conforme à la loi en cours de validité, délivrée par le représentant de l'Etat dans la région, après vérification de l'existence des moyens humains, matériels et techniques adaptés à la recherche et compatibles avec les impératifs de sécurité (article L1121-13 CSP ${ }^{[6]}$ ) ;

- les éventuelles modifications du protocole de recherche introduites à la demande du CPP ont été communiquées à l'autorité administrative compétente (article L1123-7 $\left.\operatorname{CSP}^{[6]}\right)$.

Conformément à la loi (article L1123-7 et L1243-3 CSP[6]), le Comité doit être également sollicité :

- en cas de constitution d'une collection d'échantillons biologiques (réunion, à des fins scientifiques, de prélèvements biologiques effectués sur un groupe de personnes identifiées et sélectionnées en fonction des caractéristiques cliniques ou biologiques d'un ou plusieurs membres de ce groupe) ;

- en cas d'utilisation d'éléments et de produits du corps humain à des fins scientifiques lorsqu'un changement substantiel de finalité intervient, par rapport au consentement initialement manifesté par le donneur au moment du prélèvement de ces éléments et produits.

Enfin, lorsqu'une étude visant à évaluer des soins courants est mise en œuvre, les actes étant pratiqués et les produits (à l'exception de tous ceux mentionnés à l'article L5311-1 CSP ${ }^{[6]}$ ) étant utilisés de manière habituelle, mais des modalités particulières de surveillance étant envisagées, un protocole décrivant ces modalités particulières et les conditions d'information des personnes concernées doit être rédigé et soumis pour avis préalable au CPP. La loi ne prévoit pas, en l'espèce, d'avis obligatoirement favorable.

Le rôle du CPP est particulièrement important en ce qui concerne l'information et le consentement des personnes sollicitées pour participer à une recherche : information préalable, mais également information à l'issue de l'essai. A cet égard le groupe propose une liste standard de ce que doit comporter l'information des personnes qui se prêtent à une recherche (tableau I). La loi prévoit que la personne « a le droit d'avoir communication, au cours ou à l'issue de la recherche, des informations concernant sa santé », détenues par l'investigateur, et celui d'être informée des résultats globaux de cette recherche, selon des modalités devant être précisées dans le document d'information, et dont il appartient au Comité de s'assurer de l'existence.

Lorsqu'une recherche biomédicale vise spécifiquement ou est susceptible de concerner une personne mineure ou une personne majeure sous tutelle ou sous curatelle, si le Comité considère qu'elle comporte, par l'importance des contraintes ou
Tableau I. Informations préalables au recueil du consentement

Titre, promoteur, but, objectifs, nature des produits, notion de recherche Noms et qualité des investigateurs (et collaborateurs cliniques)

Qualité des participants (nombre, caractéristiques des inclus)

Déroulement : méthode, actes, visites

Contraintes particulières à la recherche : nombre de visites, d'hospitalisations, nombre de prélèvements et quantité prélevée, examens spécifiques, durée de participation

Risques encourus/bénéfice attendu

Alternatives de traitement possibles

Suivi en fin d'essai : continuité du traitement

Délai de réflexion

Durée de l'exclusion

Inscription éventuelle sur le fichier national

Liberté de participation, droit de retrait du consentement, absence de préjudice et de responsabilité, droits maintenus

Nom et coordonnées de I 'investigateur/droit aux questions

Information et nouveau consentement si informations nouvelles

Assurance du promoteur

Indemnisations : quoi, combien et comment

Accès aux résultats globaux

Droits sur accès et rectification des fichiers CNIL

Confidentialité/anonymat des documents/publication

Personnes autorisées à consulter les données

Avis CPP

Point de contact

Circonstances ou raisons prévisibles susceptibles d'amener à interrompre la participation du sujet

CNIL = Commission Nationale Informatique et Liberté ; $\mathbf{C P P}=$ Comité de Protection des Personnes.

par la spécificité des interventions auxquelles elle conduit, un risque sérieux d'atteinte à la vie privée ou à l'intégrité du corps humain, il peut demander la mise en place des protections renforcées prévues par la loi comportant la possibilité de saisine du juge des tutelles. Il en est de même dans le cas d'une personne majeure hors d'état d'exprimer un consentement mais ne faisant pas l'objet d'une mesure de protection juridique.

La loi organisant un fichier national destiné à recenser les personnes ne présentant aucune affection se prêtant à des recherches, ou des personnes malades participant à des recherches ne concernant pas la pathologie dont elles sont porteuses, le Comité peut décider, compte tenu des risques et des contraintes que comporte une recherche, que les personnes participant à cette recherche soient recensées dans le fichier quand bien même elles n'appartiennent pas aux deux catégories ci-dessus.

Dans cette phase préalable à l'éventuelle mise en place d'un essai clinique par un promoteur, le Comité peut recevoir de 
l'autorité administrative compétente les modifications du protocole qu'elle demande au promoteur lorsqu'elle soulève des objections à la mise en place de la recherche.

Parallèlement le Comité informe l'autorité compétente des modifications au protocole qu'il a demandées.

\subsection{Rôle pendant et après l'essai}

Le CSP disposait : « Le CCPPRB peut émettre ... un avis favorable à la réalisation d'une recherche, sous réserve de la transmission d'informations complémentaires par l'investigateur pendant le déroulement de celle-ci. A la suite de cette transmission, le Comité peut maintenir ou modifier son avis ». Cette faculté n'est pas maintenue pour le CPP.

Néanmoins, en cours d'essai « toute modification substantielle de (la recherche) à l'initiative du promoteur doit obtenir, préalablement à sa mise en œuvre, un avis favorable du Comité et une autorisation de l'autorité compétente. Dans ce cas, le Comité s'assure qu'un nouveau consentement des personnes participant à la recherche est bien recueilli, si cela est nécessaire » (article L1123-9 CSP ${ }^{[6]}$ ).

De même, « les événements et les effets indésirables définis pour chaque type de recherche sont notifiés respectivement par l'investigateur au promoteur, et par le promoteur à l'autorité compétente ainsi qu'au CPP compétent. Dans ce cas, le Comité s'assure, si nécessaire, que les personnes participant à la recherche ont été informées des effets indésirables et qu'elles confirment leur consentement » (article L1123-10 CSP ${ }^{[6]}$ ).

$\mathrm{Au}$ regard de la directive européenne, ces événements et effets indésirables définis sont les événements indésirables ayant entraîné un décès, et les effets indésirables graves inattendus. La gravité s'apprécie par le fait que l'événement ou l'effet indésirable entraîne la mort, met en danger la vie, nécessite une hospitalisation ou la prolongation d'une hospitalisation, provoque un handicap ou une incapacité importants ou durables, ou bien se traduit par une anomalie ou une malformation congénitale. L'effet indésirable se différencie de l'événement indésirable par l'établissement d'une probabilité de relation de causalité entre lui et le médicament expérimental, l'événement pouvant n'être qu'un phénomène observé au cours de la recherche mais non susceptible de cette relation de causalité. Le caractère inattendu d'un effet découle du fait qu'il ne figure pas, par sa nature, sa sévérité ou son évolution dans les informations disponibles relatives au produit (par exemple, pour un médicament ne faisant pas l'objet d'une AMM, la brochure d'investigateur).

Le promoteur informe également le CPP de tout fait nouveau intéressant la recherche ou le produit faisant l'objet de la recher- che, et susceptible de porter atteinte à la sécurité des personnes qui s'y prêtent ainsi que les éventuelles mesures de sécurité prises dans une telle situation. Les usages professionnels et certaines recommandations administratives entendent, à titre d'exemple, comme fait nouveau, des observations d'effets indésirables graves et inattendus observés, pour le médicament expérimental, dans d'autres circonstances que la recherche biomédicale considérée, les résultats d'analyses intermédiaires pertinentes quant à la sécurité des personnes (notamment une efficacité insuffisante), des résultats d'études menées chez l'animal et qui apporteraient des éléments nouveaux sur la sécurité du produit, et d'une façon générale, toute information nouvelle pouvant conduire à une réévaluation dans le sens défavorable du rapport bénéfice/risque de la recherche (souligné par nous). Le «fait nouveau » peut être, d'ailleurs, en relation avec la situation de recherche et non spécifiquement avec un médicament expérimental.

La directive européenne ${ }^{[2]}$ ci-dessus évoquée dispose qu'une fois par an «et pendant toute la durée de l'essai clinique », le promoteur fournit aux Etats-membres sur le territoire desquels l'essai clinique est conduit et au comité d'éthique (terme utilisé dans les textes européens mais dont la définition officielle n'est pas incompatible avec le concept de CPP) la liste de toutes les suspicions d'effets indésirables graves survenus au cours de cette durée ainsi qu'un rapport concernant la sécurité des patients. Ce document a vocation à être transmis au CPP, même s'il n'en est pas fait mention dans la législation française.

Les modalités d'échange entre l'autorité compétente et le Comité sont à déterminer, que ce soit à propos des modifications substantielles apportées au protocole, à propos des faits nouveaux en dehors d'une situation d'urgence ou pour les effets indésirables graves (tableau II).

Considérant les missions de l'Afssaps en termes de «vigilance », le groupe, reprenant les indications détaillées européennes, ${ }^{[5]}$ propose que le Comité soit destinataire « dans les meilleurs délais » :

- des effets indésirables graves et inattendus observés en France et ceci, au cas par cas ;

- des autres effets indésirables graves, semestriellement, sous forme de tabulation, avec une analyse synthétique. Ceci afin d'informer périodiquement le Comité de la nature de ces autres effets graves inattendus relatés, ce dès lors que l'analyse d'imputabilité est réalisée, ce qui implique une levée de l'anonymat dans les essais comparatifs, acte considéré comme discutable par la plupart des méthodologistes, en cours d'essai, sauf précaution particulière. 
Tableau II. Missions Afssaps - CPP et échanges d'informations en cours d'essai

\begin{tabular}{ll}
\hline Propositions & \\
\hline $\begin{array}{l}\text { Vigilance : Rôle de l'Afssaps } \\
\text { et du CPP }\end{array}$ & $\begin{array}{l}\text { L'Afssaps définit la stratégie générale et dans le cas des « faits nouveaux » les principes généraux qui doivent être mis } \\
\text { en application } \\
\text { Le CPP avec le promoteur organisent la déclinaison dans les différentes études }\end{array}$ \\
$\begin{array}{ll}\text { Echange d'informations } \\
\text { Afssaps - CPP - Promoteur } \\
\text { amendements }\end{array}$ & $\begin{array}{l}\text { Même mode de fonctionnement que pour le dossier initial (soumission parallèle), contact direct CPP-Afssaps avec copie } \\
\text { promoteur }\end{array}$ \\
& "Coordination » Afssaps d'une réponse synthétique au promoteur à condition de respecter les délais initiaux des 35 \\
& jours $\rightarrow$ question du délai des CPP et possibilité d'évaluations divergentes entre CPP et Afssaps \\
Transmission de l'information en parallèle aux CPP et à l'Afssaps (autre possibilité : transmission Afssaps vers \\
promoteur copie au CPP) \\
L'Afssaps si nécessaire définit les principes généraux devant être mis en œuvre et discute avec le promoteur, le CPP \\
contrôle la mise en œuvre par le promoteur (nouvelle note d'information, amendements ...) Quid en cas de contestation \\
de la demande de modification ? \\
Recommandation de listings semestriels \\
Déclaration pour le comparateur?
\end{tabular}

Afssaps = Agence française de sécurité sanitaire des produits de santé $; \mathbf{C P P}=$ Comité de Protection des Personnes $;$ SUSAR = suspected unexpected serious adverse reaction.

Il convient de différencier événement et effet, l'événement se caractérise par sa seule survenue, l'effet est un événement pour lequel une relation de causalité avec la recherche ou un médicament a été établie.

Une remarque à ce sujet : la directive européenne ${ }^{[2]}$ ci-dessus évoquée retient le principe de la relation de causalité avec le médicament expérimental, la législation française se préoccupe, elle, de la relation de causalité avec la situation de recherche.

Enfin, le Comité doit être informé (dans des délais à préciser réglementairement), soit de la fin de l'essai intervenant au terme et dans les conditions prévues, soit des raisons motivant un arrêt anticipé.

\subsection{Responsabilité du CPP}

Selon l'analyse faite par les juristes composant le sousgroupe 3 (Joël Moret-Bailly, Vincent Diebolt, Thomas Roche), il apparaît que le rôle et la définition de la nature des responsabilités des CPP ne peut s'effectuer qu'après une remise en perspective. Les règles européennes relatives aux « comités d'éthique », leur transposition en droit français sous la forme de « comités de protection des personnes » ne sauraient conduire à la réplication, en Europe, comme en France des Institutional Review Boards rencontrés aux Etats-Unis.

En outre, chaque concept juridique s'inscrit et s'interprète dans le cadre du système auquel il appartient. Le Comité de protection relève du «droit administratif » (concept ignoré, par exemple, dans le droit anglosaxon). L'avis du CPP, prévu par la loi du 9 août $2004,{ }^{[3]}$ intégrée au $\mathrm{CSP},{ }^{[6]}$ relève des règles générales relatives à la procédure des avis consultatifs de l'administration.

Rappelons que le droit, lorsqu'il ne crée pas ses propres concepts, donne aux mots un sens particulier, fonction de sa logique propre et des buts qu'il poursuit. Il y a par conséquent toutes les chances que les notions d' « avis », de «validité » et de «pertinence », que l'on retrouve dans la loi aux passages relatifs aux missions des Comités, diffèrent, pour les juristes, du sens qu'on peut leur donner dans le langage courant, voire dans celui des professionnels de la recherche biomédicale. Or, c'est à l'aune du droit qu'une loi s'interprète.

Nombre de décisions administratives sont prises suite à la consultation d'autorités compétentes chargées de donner leur avis sur une question technique, voire sur la pertinence de la décision envisagée. Dans ce contexte, une définition « élémentaire » et couramment admise chez les juristes admet que l'avis est un terme «s'appliquant dans toutes les branches du droit au résultat de consultations, facultatives ou obligatoires selon le cas, demandées aux organes les plus divers (personnes ou commissions, conseils, fonctionnaires qualifiés, Conseil d'Etat, etc.) ». ${ }^{[7]} \mathrm{Il}$ existe, dans ce contexte, trois types d'avis administratifs : les avis simples, les avis obligatoires, et les avis conformes.

Les premiers, les avis simples, sont laissés à la discrétion de l'autorité administrative, qui peut les solliciter ou non, sans incidence sur la régularité de sa décision. Les seconds, les avis obligatoires, sont, comme leur nom l'indique, impératifs pour l'autorité décisionnelle, en ce qu'elle ne peut régulièrement s'en 
passer, mais sans que cette dernière soit liée par le sens de l'avis. Autrement dit, elle peut décider dans un sens contraire à celui de l'avis, sans conséquence quant à la légalité de sa décision. Le troisième type d'avis, l'avis conforme, est non seulement obligatoire, mais lie l'autorité administrative quant au sens de sa décision. Celle-ci ne dispose donc plus que d'une alternative : décider dans le sens de l'avis, ou renoncer à prendre la décision projetée. ${ }^{[8]}$

Dans ce contexte, l'avis des CPP appartient à la troisième catégorie : les avis conformes. La directive du 4 avril 2001[2] prévoyait, en effet, dans son article 6 , que le comité « est tenu d'émettre son avis avant le commencement de tout essai clinique au sujet duquel il a été sollicité », ce qui le rend obligatoire. Mais elle prévoyait également, dans son article 9, que « le promoteur ne peut commencer un essai clinique qu'après délivrance d'un avis favorable de la part du Comité ». L'article 88 de la loi du 9 août, ${ }^{[3]}$ codifié dans l'article L1121-4 $\mathrm{CSP}^{[6]}$ transpose cette norme en prévoyant que « la recherche biomédicale ne peut être mise en œuvre qu'après avis favorable du comité de protection des personnes (...) et autorisation de l'autorité compétente ». Un point à relever cependant. La directive impose un délai de 60 jours maximum au Comité pour communiquer son avis au promoteur et à l'autorité compétente. Mais elle prévoit que l'autorité compétente peut néanmoins notifier au promoteur, avant la fin de cette période, qu'elle n'a pas d'obstacle à l'autorisation de l'essai. La directive dispose également que l'autorité compétente peut « si cela est conforme à la pratique habituelle » examiner une demande d'autorisation en un délai inférieur à ces 60 jours. ${ }^{[2]}$ Il en résulte que l'autorité compétente peut choisir de prendre sa décision d'autorisation sans connaître l'avis du Comité. Ce, selon la règle communautaire.

Les enjeux de cette qualification de l'avis au regard du droit administratif sont d'importance quant à la régularité de la recherche. Le rôle de l'administration réside, en effet, tant politiquement que juridiquement en droit français, et en tant qu'émanation du Pouvoir exécutif, dans l'application de la loi, incarnation, quant à elle, de la volonté générale, ni plus ni moins, même si cette fonction peut la conduire elle-même à produire des normes juridiques d'application. L'administration ne peut, dans une telle perspective politique, qu'être placée sous le contrôle des citoyens, puisque ces derniers sont à l'origine de la loi. On comprend donc que les décisions administratives (et les avis conformes leur sont assimilés) puissent faire l'objet d'un recours, destiné à anéantir l'acte illégal. Il s'agit du « recours pour excès de pouvoir », exercé devant les juridictions administratives (tribunal administratif, cour administrative d'appel et Conseil d'Etat) qui aboutit, s'il est fondé, à l'annulation de la décision administrative qui est alors censée n'avoir jamais existé. L'intérêt du recours, destiné à défendre la légalité, est même tel qu'il est ouvert à toutes les personnes intéressées par la décision, personnes physiques ou morales, autres administrations, contribuables, etc.

On peut affirmer, in fine, que la non-obtention d'un avis favorable du CPP rend la recherche illégale et que « l'autorité compétente » (Afssaps) ne peut autoriser une recherche ayant fait l'objet d'un avis défavorable du CPP. Cette dernière peut, en revanche, refuser l'autorisation à une recherche ayant bénéficié d'un avis favorable, notamment pour des raisons de police sanitaire. Elle peut, réciproquement, former un recours pour excès de pouvoir contre l'avis défavorable d'un CPP.

Reste, cependant, à connaître l'étendue du contrôle des CPP sur les protocoles de recherche, notamment en ce qui concerne leur «validité » et leur «pertinence », puisque la loi leur en confie la mission.

L'article L1123-7 CSP ${ }^{6]}$ prévoit, depuis le 9 août 2004, que « le Comité rend son avis sur les conditions de validité de la recherche, notamment au regard de : la protection des personnes, notamment la protection des participants ; l'adéquation, l'exhaustivité et l'intelligibilité des informations écrites à fournir ainsi que la procédure à suivre pour obtenir le consentement éclairé, et la justification de la recherche sur des personnes incapables de donner leur consentement éclairé ; la nécessité éventuelle d'un délai de réflexion ; la nécessité éventuelle de prévoir, dans le protocole, une interdiction de participer simultanément à une autre recherche ou une période d'exclusion ; la pertinence de la recherche, le caractère satisfaisant de l'évaluation des bénéfices et des risques attendus (évaluation réalisée par le promoteur de la recherche) et le bien-fondé des conclusions de cette évaluation ; l'adéquation entre les objectifs poursuivis et les moyens mis en œuvre ; la qualification du ou des investigateurs ; les montants et les modalités d'indemnisation des participants ; les modalités de recrutement des participants » (c'est nous qui soulignons).

Dans ce contexte, le concept de « validité de la recherche » ne pose pas de réelles difficultés juridiques. En effet, le terme « validité » revêt, en droit, un sens tout à fait précis : il s'agit de l'absence de contrariété avec les normes hiérarchiquement supérieures. Il s'agit donc, ici, de contrôler la non-contrariété entre les conditions de la recherche et les règles légales. C'est d'ailleurs bien à ces dernières que fait référence la suite du texte. La mission du Comité réside donc dans le fait de rendre un avis sur la question suivante : les conditions de la recherche permettentelles le respect de la loi, donc la protection des personnes?

Dans cette perspective, seule la question de la pertinence scientifique de la recherche se révèle délicate. La loi prévoit, en 
effet, que le Comité exerce sa mission en ce qui concerne «la pertinence de la recherche, le caractère satisfaisant de l'évaluation des bénéfices et des risques attendus et le bien-fondé des conclusions, l'adéquation entre les objectifs poursuivis et les moyens mis en œuvre » (c'est nous qui soulignons). Le Comité se transforme-t-il, de ce fait, en comité d'experts, chargé d'apprécier la pertinence scientifique de la démarche ? La réponse (très débattue au sein de la table ronde) est sans doute négative. En effet, outre l'argument précédent, selon lequel la validité, dans un énoncé juridique, doit s'entendre comme la noncontrariété avec une norme juridique supérieure (et non, par exemple, une norme scientifique, dont on ne voit pas, de surcroît, ce qu'elle pourrait avoir d'inférieur ou de supérieur à une règle de droit, n'étant pas de même nature), on peut douter que, dans les faits, un CPP puisse accueillir toutes les compétences nécessaires pour couvrir l'ensemble des domaines de recherche, et ce dans leurs dimensions scientifiquement les plus pointues. Pragmatiquement, voire prosaïquement, les CPP ne peuvent sans doute pas être des comités d'experts, bien que la tentation soit grande, du fait de la présence, au sein du Comité, de professionnels de la recherche biomédicale. Rien n'empêche toutefois les Comités de pouvoir faire appel à des experts scientifiques ou autres pour éclairer leurs débats.

On peut avancer, pour achever de se convaincre, des arguments comparatifs. Nous prendrons, pour ce faire, l'exemple de l'indemnisation des accidents médicaux, des infections nosocomiales et de l'alea thérapeutique telle qu'organisée par la loi 2002-303 du 4 mars 2002 ${ }^{[9]}$ relative aux Droits des personnes malades et des usagers du système de santé. Celle-ci crée notamment, à cet effet, des commissions régionales d'indemnisation des accidents médicaux (CRCI) chargées de fournir un avis sur l'origine du dommage devant être indemnisé à l'office national d'indemnisation (ONIAM). Les CRCI comprennent, notamment, du fait de la décision du législateur, des professionnels de santé. Elles ne constituent cependant absolument pas des instances expertales. La preuve en est qu'elles ne rendent leurs avis que suite à expertise. Pourquoi, dès lors, intégrer des professionnels de santé à ces structures ?

Outre l'aspect politiquement sensible de la question, qui conduit à associer les professionnels au processus, il s'agit de profiter de leur aptitude à comprendre, mieux que d'autres, les informations en cause, notamment telles qu'elles résultent justement des rapports d'expertise.

Il convient de raisonner de même en ce qui concerne les CPP. Les professionnels de la recherche figurent dans les CPP (outre pour des raisons historiques - les premiers « comités d'éthique » étant nés à l'initiative de professionnels et composés de ces derniers), ${ }^{[10]}$ du fait de leurs compétences leur permettant de comprendre les protocoles présentés. Autrement dit, il ne s'agit pas, pour les CPP, d'opérer un contrôle scientifique approfondi des protocoles de recherche (apanage de «l'autorité compétente », chargée, en ce qui la concerne, d'une mission de police sanitaire et disposant de moyens lui permettant de recouper nombre d'informations, ainsi que de faire appel, le cas échéant, à des experts), mais de vérifier que ceux-ci ne violent pas la loi (et, sur un plan scientifique, sans doute de manière flagrante pour un homme de l'art) eu égard au but même de cette dernière, inspirant la dénomination des comités : la protection des personnes.

Deux articles relatifs à la responsabilité des Comités méritent d'être individualisés :

- en cas d'avis défavorable d'un Comité, le promoteur peut demander au ministre chargé de la santé de soumettre le projet de recherche, pour un second examen, à un autre Comité selon des conditions définies par voie réglementaire (article L1123-6 CSP ${ }^{6]}$ ) ;

- en cas de faute du Comité dans l'exercice de sa mission, la responsabilité de l'Etat est engagée (article L1123-8 CSP ${ }^{[6]}$ ).

\subsection{Organisation des CPP}

\subsection{Constat}

La loi du 9 août $2004^{[3]}$ a introduit des données nouvelles concernant le CPP au regard de son prédécesseur le CCPPRB.

Article L1123-1 CSP :[6]

«Le ministre chargé de la santé agrée au niveau régional pour une durée déterminée un ou, selon les besoins, plusieurs Comités de protection des personnes et détermine leur compétence territoriale. Leurs membres sont nommés par le représentant de l'Etat dans la région.

Les Comités exercent leur mission en toute indépendance. Ils sont dotés de la personnalité juridique. »

Article L1123-2 CSP :[6]

«Les Comités sont composés de manière à garantir leur indépendance et la diversité des compétences dans le domaine biomédical et à l'égard des questions éthiques, sociales, psychologiques et juridiques. Ils comportent, en leur sein, des représentants d'associations de malades ou d'usagers du système de santé agréées et désignés. »

Article L1123-3 CSP :[6]

«Les membres des Comités, les personnes appelées à collaborer à leurs travaux, et les agents relevant du statut général des fonctionnaires qui en sont dépositaires sont tenus, dans les conditions et sous les peines prévues aux articles 226-13 et 22614 du code pénal, de garder secrètes les informations dont ils peuvent avoir connaissance à raison de leurs fonctions et qui sont 
relatives à la nature des recherches, aux personnes qui les organisent ou qui s'y prêtent ou aux produits, objets ou méthodes expérimentés.

Les membres du Comité adressent au représentant de l'Etat dans la région, à l'occasion de leur nomination, une déclaration mentionnant leurs liens, directs ou indirects, avec les promoteurs et les investigateurs de recherches. Cette déclaration est rendue publique et actualisée à leur initiative dès qu'une modification intervient concernant ces liens ou que de nouveaux liens sont noués.

Ne peuvent valablement participer à une délibération les personnes qui ne sont pas indépendantes du promoteur et de l'investigateur de la recherche examinée. »

\section{Article L1123-4 CSP :[6]}

«Les frais de fonctionnement des Comités sont financés par le produit d'un droit fixe versé par les promoteurs pour chacun des projets de recherches biomédicales faisant l'objet d'une demande d'avis. Le montant de ce droit est arrêté par le ministre chargé de la santé. »

Article L1123-5 CSP :[6]

«Le ministre chargé de la santé peut retirer l'agrément du Comité si les conditions d'indépendance, de composition ou de fonctionnement nécessaires pour assurer sa mission dans les meilleures conditions ne sont plus satisfaites. »

Les modalités d'application des dispositions citées ci-dessus seront déterminées par décret en Conseil d'Etat, en cours de rédaction au moment de la table ronde.

C'est le cas tout particulièrement de :

Article L1123-14 CSP :[6]

« $1^{\circ} \mathrm{La}$ composition et les conditions d'agrément, de financement, de fonctionnement et de nomination des membres des Comités de protection des personnes ainsi que la nature des informations qui doivent leur être communiquées par le promoteur et sur lesquelles ils sont appelés à émettre leur avis ;

$2^{\circ}$ La durée des agréments des Comités de protection des personnes ;

$3^{\circ} \mathrm{La}$ nature des informations qui doivent être communiquées par le promoteur à l'autorité compétente, dans la demande d'autorisation mentionnée à l'article L. 1121-4 ;

$5^{\circ}$ Les modalités de présentation et le contenu de la demande de modification de la recherche prévue par l'article L. 1123-9;

$7^{\circ}$ La nature et le caractère de gravité des événements et des effets indésirables qui sont notifiés ainsi que les modalités de cette notification ;

$8^{\circ}$ Les modalités selon lesquelles le promoteur informe l'autorité compétente et le Comité de protection des personnes de l'arrêt de la recherche ; $9^{\circ}$ Les modalités d'évaluation prévues sur la base du référentiel d'évaluation des Comités de protection des personnes élaboré par l'Agence nationale d'accréditation et d'évaluation en santé et publié par arrêté du ministre chargé de la santé ;

$11^{\circ}$ Les délais dans lesquels le Comité rend l'avis mentionné à l'article L. 1123-7 et l'autorité compétente délivre l'autorisation mentionnée à l'article L. 1123-8. »

L'expérience acquise grâce aux CCPPRB conduit à souhaiter un renforcement de certains aspects administratifs du fonctionnement, ce d'autant que le Comité a le caractère de personne de droit public, que le caractère obligatoirement favorable de son avis, s'incluant dans une décision d'autorisation est susceptible de recours, y compris de recours fondés sur la façon dont un avis défavorable a été rendu.

On a pu constater, dans le système antérieur, de notables faiblesses inhérentes aux difficultés de recrutement de bénévoles pour participer aux séances, à la mauvaise information des personnes sollicitées pour s'intégrer à un CCPPRB (tant sur la structure que sur leur rôle éventuel), à l'hétérogénéité de la formation des nouveaux membres, tout ceci se traduisant à terme par une baisse de l'assiduité aux réunions.

D'un Comité à l'autre, d'une région à l'autre, existent des écarts significatifs sur le nombre de dossiers soumis à avis, de la charge de travail, et une hétérogénéité dans les processus d'évaluation et les avis rendus.

\subsubsection{Propositions}

Le groupe propose les principaux éléments devant permettre un fonctionnement harmonieux des CPP (tableau III).

\section{Commentaires}

L'habituelle différence d'interprétation entre les professionnels de l'essai clinique et les juristes quant aux missions des Comités n'a pas manqué d'être observée au cours des débats, notamment quant au sens des termes «validité » et «pertinence » de la recherche. De la même façon a-t-on pu s'interroger sur l'implication du Comité dans l'appréciation de la qualification des investigateurs, l'adéquation du lieu aux conditions de la recherche et le caractère satisfaisant de l'évaluation du « rapport bénéfice/risque » de la recherche. Le législateur ayant disposé parallèlement le Comité de protection et l'autorité administrative, la question s'est posée de savoir si les rôles respectifs des deux les conduisaient à la complémentarité ou au recoupement. Il apparaissait de toute façon nécessaire que les échanges entre l'autorité compétente et le Comité, échanges susceptibles d'être bilatéraux, soient organisés ne serait-ce que pour réduire au maximum les délais nécessaires à l'obtention d'un avis et d'une 
Tableau III. Mettre en place un fonctionnement harmonieux CPP

Fonctionnement harmonisé commun à tous les CPP (fixation par
décret/arrêté)
Règlement intérieur
Procédures
Modalité d'appel à candidature des membres, et composition du groupe
(médicaux/non-médicaux)
Modalité de recours aux experts
Formation des membres
Audit et inspection
Déclaration des conflits d'intérêts
Traçabilité documentaire et budgétaire
Relations avec la DGS, l'Afssaps, la DRASS
Relations avec les associations de patients, le grand public
Indemnisation des membres et rémunération des experts

\section{Développement du réseau des CPP}

Affiliation obligatoire au réseau (décret?)

Développement de Bonnes Pratiques et principe d'une démarche de qualité

Propositions pour l'amélioration du fonctionnement

Support informatique pour favoriser la permanence des échanges

Etudes de cas, formation, etc.

Afssaps = Agence française de sécurité sanitaire des produits de santé ;
CPP = Comité de Protection des Personnes ; DGS = Direction Générale
de la Santé ; DRASS = Direction Régionale de l'Action Sanitaire et Sociale.

autorisation. Il existe effectivement entres les Etats-membres une compétition en termes d'attractivité pour les promoteurs de recherches cliniques, jouant en particulier sur la durée plus ou moins courte des « temps administratifs ».

L'introduction du système français dans cette concurrence européenne a paru devoir se traduire, pour les participants à la table ronde, par la mise en œuvre de moyens nouveaux. Ces moyens pourraient être un meilleur financement des coûts de fonctionnement (locaux, secrétariat, outils, archivage), et la création d'un budget relatif aux frais de personnel, comportant la possibilité de rétribuer des experts extérieurs, et d'indemniser les membres du Comité des coûts induits par leur participation aux séances, notamment les frais de déplacements, voire le manque à gagner.

Du point de vue structurel, le caractère de personne de droit public du Comité a conduit à insister sur la nécessité d'un statut, d'un règlement intérieur, et de la rédaction de procédures standard de fonctionnement, accessibles et dont le respect serait vérifiable.

La présence de membres d'associations de malades ou d'usagers du système de santé au sein des CPP constitue un atout important en termes «d'intelligibilité des informations » fournies aux personnes sollicitées pour participer à une recherche.

Sans pour autant affecter l'indépendance de règle des Comités, il a été souhaité une coordination de ceux-ci à l'échelon national, avec des procédures destinées à unifier le fonctionnement et la fiabilité de l'évaluation, une structure d'information et de coordination, aussi bien des Comités eux-mêmes que des membres. Concernant ces derniers, le souhait de leur information et de leur formation, aux objectifs, responsabilités et fonctionnement de la structure elle-même a pu être émis. Compte tenu de l'existence de leur part d'une déclaration actualisée relative aux éventuels conflits d'intérêt qu'ils pourraient présenter, il a été souhaité que celle-ci fasse l'objet d'une définition de ses modalités.

Enfin les membres de la table ronde se sont accordés pour proposer l'élaboration d'un référentiel permettant l'évaluation des pratiques des Comités, afin de répondre au souci d'excellence, présent dans les esprits, et de favoriser le professionnalisme et la stabilité des Comités, pour préserver sur le long terme les expériences acquises.

\section{Participants}

P. Amiel (Université Paris-8, Paris), F. Berger (Aventis, Paris), C. Bernaud (Sankyo Pharma, Paris), N. Best (DRRC, Paris), I. Brindel (AP-HP, Paris), F. Chapuis (CCPPRB, Lyon), M. Couderc (Sanofi-Synthelabo, Paris), S. Courcier Duplantier (Roche, Neuilly-sur-Seine), F. De Crémiers (Wyeth, Paris La Défense), A. Deneulin (AstraZeneca, Rueil-Malmaison), V. Diebolt (Féd. Hospitalière de France, Paris), T. Dupin Spriet (Fac Pharmacie, Lille), C. Funck Brentano (Hôpital St-Antoine, Paris), J. Genève (BECT/FNCLCC, Paris), C. Guérin (CCPPRB Cochin, Paris), A. Guillardeau Jaillon (Hôpital St-Antoine, Paris), S. Hansel (CCPPRB, Montpellier), S. Hilaly (AP-HP, Paris), P. Lechat (CCPPRB Pitié-Salpétrière, Paris), F. Lemaire (Hôpital H. Mondor, Créteil), F. Loeb (DRASSIF, Paris), G. Loeb (Yamanouchi Pharma, Paris La Défense), A. Mijonnet (Merck-Sharp \& Dhome Chibret, Paris), J. Moret Bailly (Univ. Saint-Etienne), C. Nourissier (Eurordis - plate-forme maladies rares, Versailles), C. Oréfice (3M Santé, Cergy-Pontoise), J.-M. Laurent Vo (DGS, Paris), Y. Plétan (Pfizer, Paris), T. Roche (Cab. Avocats, Lyon), P. Sassano (Novartis Pharma, Rueil-Malmaison), C. Sibenaler (LEEM, Paris), B. White Gay (Merck \& Co., USA).

\section{Références}

1. Loi 88-1138 du 20 Décembre 1988 loi relative à la protection des personnes qui se prêtent à des recherches biomédicales [online]. Available from URL: http//www.legifrance.gouv.fr/ [Accessed 2005 Jul 11]

2. Directive 2001/20/CE of the European Parliament and of the Council of 4 April 2001 on the approximation of the laws, regulations and administrative provisions of the Member States relating to the implementation of good clinical practice in the conduct of clinical trials on medicinal products for human use, 2001 May OJ 1 (L 121) 34-44

3. Loi n ${ }^{\circ}$ 2004-806 du 9 août 2004 loi relative à la politique de santé publique [online]. Available from URL: http//www.legifrance.gouv.fr/ [Accessed 2005 Jul 14]

4. European Commission. Detailed guidance for the request for authorisation of a clinical trial on a medicinal product for human use to the competent authorities, 
notification of substantial amendments and declaration of the end of the trial. April 2004. Brussels: European Commission, 2004

5. European Commission. Detailed guidance on the application format and documentation to be submitted in an application for an Ethics Committee opinion on the clinical trial on a medicinal product for human use. April 2004. Brussels: European Commission, 2004

6. Code de la santé publique [online]. Available from URL: http//www.legifrance.gouv.fr/ [Accessed $2005 \mathrm{Jul} 11]$

7. Guillien R, Vincent J, Guinchard S, et al. Lexique des termes juridiques. Paris: Dalloz, 2003

8. Gaudemet Y. Traité de droit administratif. Tome 1 : Droit administratif général. 16ème édition. Paris: LGDJ, 2001: 127, 128
9. Loi n ${ }^{\circ} 2002-303$ du 4 mars 2002 relative aux droits des malades et à la qualité du système de santé [online]. Available from URL: http//www.legifrance.gouv.fr/ [Accessed 2005 Jul 14]

10. Thouvenin D. Les lois bioéthiques ou comment masquer les intérêts contradictoires. In: La bioéthique est-elle de mauvaise foi? Paris: PUF, 1999: $50-78$

Correspondance et offprints : Jean-Paul Demarez, Pierre Fabre Médicament, Direction Sécurité du Médicament, 45 place Abel Gance, 92654 Boulogne Cedex, France.

E-mail : jean.paul.demarez@pierre-fabre.com 